\title{
Vestibular Rehabilitation - Recommended Treatment for Permanent Unilateral Vestibular Loss
}

\section{Mădălina Georgescu1,2*}

1 University of Medicine and Pharmacy 'Carol Davila' Bucharest, Romania

${ }^{2}$ Audiology and Vestibulogy Department, Institute of Phono-Audiology and Functional ENT Surgery Bucharest, Romania

Equilibrium is a complex network, based on several sensorial information - somatosensorial, visual and vestibular. Normal input from all three sensorial systems as well as matched sensorial information are required for normal human balance.

Vestibular sensorial structures are in the inner ear, in the posterior labyrinth and they are responsible for detecting any angular and linear acceleration of head and body. In response to movement, effects of gravity and the position in space of the head and body, relatively to ground, vestibular sensorial hair cells are stimulated and action potentials originated here are send to the nervous central system.

Any disruption in normal functioning of one of the vestibular sensorial structures leads to an asymmetry between the two vestibular pathways, which are interpreted as rotation. As consequence of this vestibular nuclei activity asymmetry, spinning sensation and nystagmus appears.

Acute unilateral vestibular loss (UVL) induces severe static and dynamic deficits, with long lasting effects on balance, thus reducing patients' quality of life. Lesions in the inner ear (viral or bacterial labyrinthitis) or vestibular ganglia or nerve (vestibular neuritis, zoster oticus) induce permanent vestibular deficit. Neural lesions are much more difficult to recover and this occurs due to vestibular compensation, not due to reversible lesions. Viral infection of the vestibular sensorial system, known as vestibular neuritis is the most common cause of UVL and the second most common cause of peripheral vestibular syndrome. It is a pathological condition that usually affects active, healthy, middleaged people. Worsening the health-related quality of life (HRQoL) in this category of people has a negative impact on their social life and work performance, leading not only to psychological damage (low selfconfidence, depression, frustration), but also economical losses (long medical leave, poor concentration and performance).

Vestibular neuritis it is also known as vestibular neuronitis, acute labyrinthitis, unilateral acute vestibular palsy, epidemic vertigo. It is caused by reactivation of a retrovirus (herpes simplex type I) which determines degenerative lesions in the vestibular nerve, vestibular ganglia or/and vestibular hair cells. It usually appears in viral infection context, usually in spring and autumn, with "epidemic" character. Hearing is never affected in vestibular neuritis $[1,2]$.

Vestibular neuritis is clinically expressed as long-lasting vertigo (more than $24 \mathrm{~h}$ ), nausea, vomiting and severe gait impairment, caused by static deficits (lesions of the vestibular structures). These symptoms have a sudden onset and high severity from the very beginning of the disease, but resolves by themselves in three to five days from the onset. History, together with bed-side vestibular examination, head impulse test (clinical or video head impulse test - vHIT) [3] and pure tone audiometry allow positive diagnostic of vestibular neuritis (Figure 1).

More important for patient's well-being are the dynamic deficits which occur in the first week. Reduced gain of vestibulo-ocular and vestibulo-spinal reflexes impedes on patient's capacity to perform daily activities. After the acute stage, vestibular objective tests are required, in order to quantify vestibular deficit (posturography, rotatory and caloric test in videonystagmography and vestibular evoked myogenic potentials - VEMP) (Figure 2).
Viral infection can affect one or both branches of the vestibular nerve, superior and inferior, causing a partial or complete vestibular neuritis. Usually, the superior vestibular branch is affected and this leads to caloric hypo- or areflexia on the damaged side. Cervical VEMP (cVEMP) is recommended for inferior vestibular nerve branch evaluation.

It is important to know the extension of the vestibular impairment in order to quantify the deficit and appropriate customise the vestibular rehabilitation program. Another reason for evaluating both superior and inferior vestibular nerve branches is to council the patient regarding long-term evolution of the disease. When a partial neuritis occurs, any lesion on the posterior semi-circular canal or saccule will become symptomatic, since information from these sensorial structures will be send through the normal functioning inferior vestibular nerve. BPPV is the most common complication of a partial vestibular neuritis and patients should be aware of this.

Natural recovery mechanism is based on central vestibular compensation, which reduces vestibular activity on the healthy part and increase vestibular activity on the lesion side, by opening closed synapses and stimulating new formation of neural sprouts (Figure 3).

Central compensation is a neuroplasticity model of recovery after a UVL. This mechanism has its highest intensity one week after vestibular injury and continues slowly on a long period of time (one year). For this reason, vestibular suppressant treatment recommended during acute onset of the vestibular neuritis should not exceed three days of administration [4].

In order to obtain a maximum gain recovery in a short period of time, central compensation should be facilitated through longterm administration of betahistine and vestibular rehabilitation (VR) programs. VR is a physical treatment which allows recovery of the balance deficits by creating new patterns of reaction to different daily balance situation.

This combined treatment protocol of UVL patients is widely accepted [5-7] for a faster and more complete recovery of the unilateral vestibular loss. VR is based on specific programs of physical exercises that can diminish the negative effects of the vestibular impairment. VR sessions are recommended weekly.

VR exercises target a reset of the brain through habituation

*Corresponding author: Mădălina Georgescu, MD, PhD, Associate Professor, University of Medicine and Pharmacy 'Carol Davila' Bucharest, Romania, Tel: +40722544115 E-mail: madalina.georgescu@gecad.com; madalina.georgescu@otomed-center.ro

Received July 11, 2017; Accepted August 03, 2017; Published August 10, 2017

Citation: Georgescu M (2017) Vestibular Rehabilitation - Recommended Treatment for Permanent Unilateral Vestibular Loss. Int J Neurorehabilitation 4: 282. doi: 10.4172/2376-0281.1000282

Copyright: @ 2017 Georgescu M. This is an open-access article distributed under the terms of the Creative Commons Attribution License, which permits unrestricted use, distribution, and reproduction in any medium, provided the original author and source are credited. 
(reduces avoidance of certain positions), adaptation (teaching the unaffected balance receptors to undertake the function of destroyed ones) and substitution (teaching other sensory systems to compensate for the vestibular impairment) strategies. The treatment is focused on improving clear vision when moving the head, reducing the intolerance to movement by use of repetitive eye, head and body movement and relearning of balance.

Customised VR program includes daily exercise sessions at home (derived from first original since 1946 Cawthorne's [5], Cooksey's [6] and Herdman's [7] exercises) and weekly sessions on special equipment, dedicated for this physical recovery - NEUROCOM platform, for example. At home exercises are customised according to the patient complaints and improvement of symptoms. Platform based exercises are selected based on patient's preference on visual or somatosensorial cues. These weekly sessions comprised exercises of weight shifting and mobility on a powered platform providing visual feedback of the centre of gravity and adjustable surface and surround.
The efficacy of the used treatment can be measured both objectively - the SOT test (sensory organisation test of the posturography) and subjectively by means of DGI (dynamic gait index), DHI (dizziness handicap inventory) questionnaire and the $\mathrm{ABC}$ scale (activitiesspecific balance confidence scale). The SOT allows evaluation of the tested subject's capacity of maintaining his balance in situations similar to daily activities - the result is shown as percentage of stability, thus sustaining the quantification of the patient's evolution under treatment. DGI is a reliable test developed to assess falling risk while walking in older adults. It consists in tasks evaluating gait in 8 challenging situations: normal speed walking for 20 feet distance, change in gait speed, gait with head turns (horizontal/vertical), gait and pivot turning, stepping over/around obstacle, walking up stairs. Maximum score is 24 points and scores of 19 or less have been related to increase incidence of falls in the elderly. DHI is a validated questionnaire for auto-appreciation of the quality of life in patients with vestibular problems. It consists of 25 standard questions, grouped

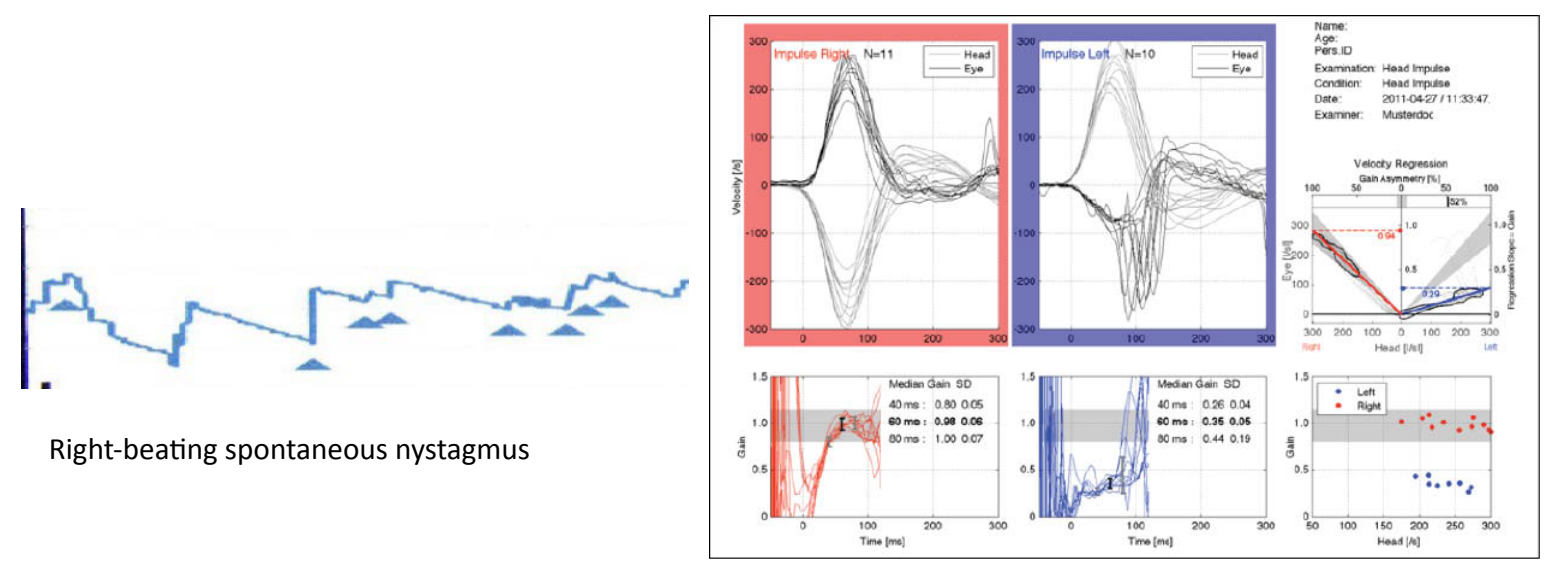

Positive vHIT on left side (gain<0.7, "overt" and "covert" saccades)

Figure 1: Acute vestibular neuritis findings.
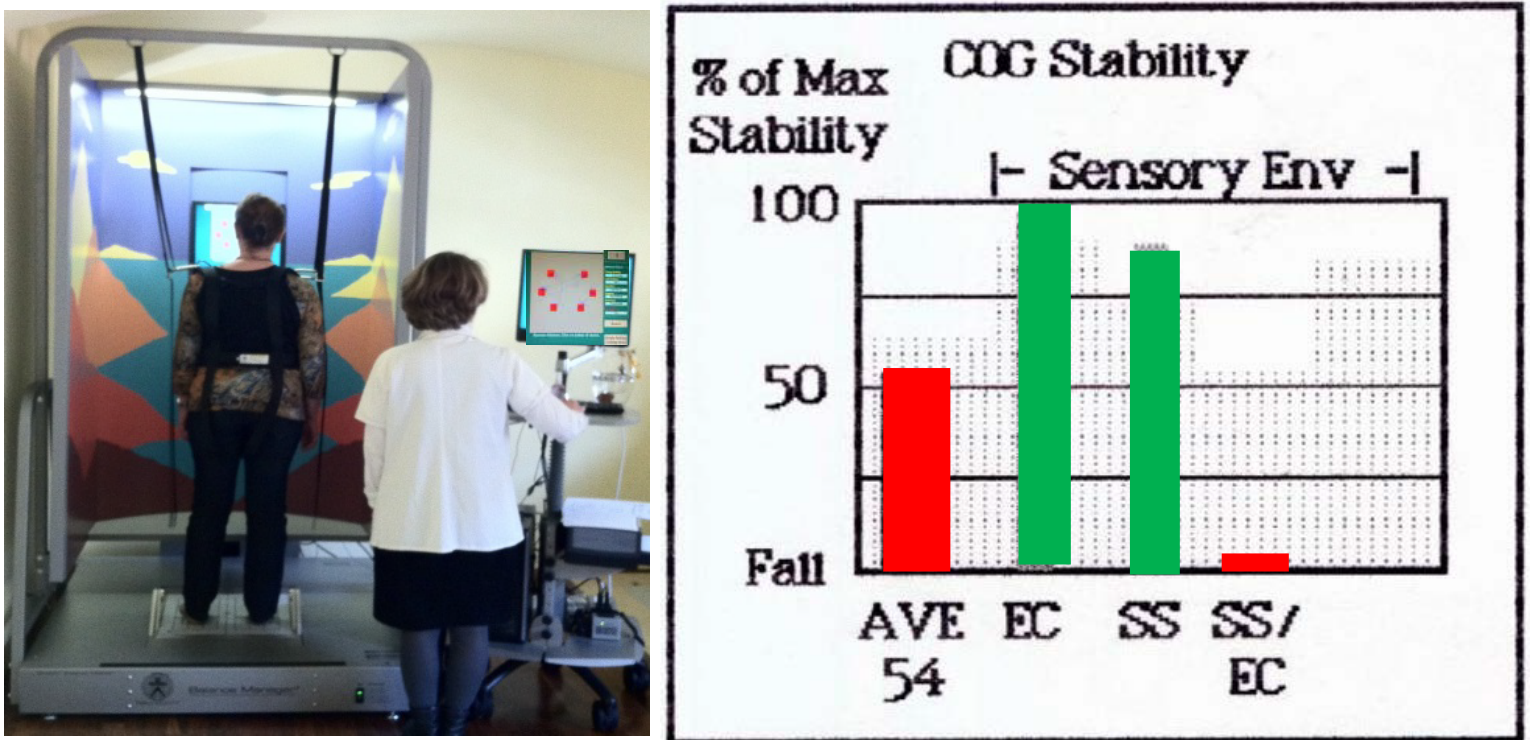

Figure 2a: Objective vestibular test - posturography (SMART Balance Master ${ }^{\circledR}$ Neurocom system) vestibular pattern (0\% stability score in vestibular condition). 

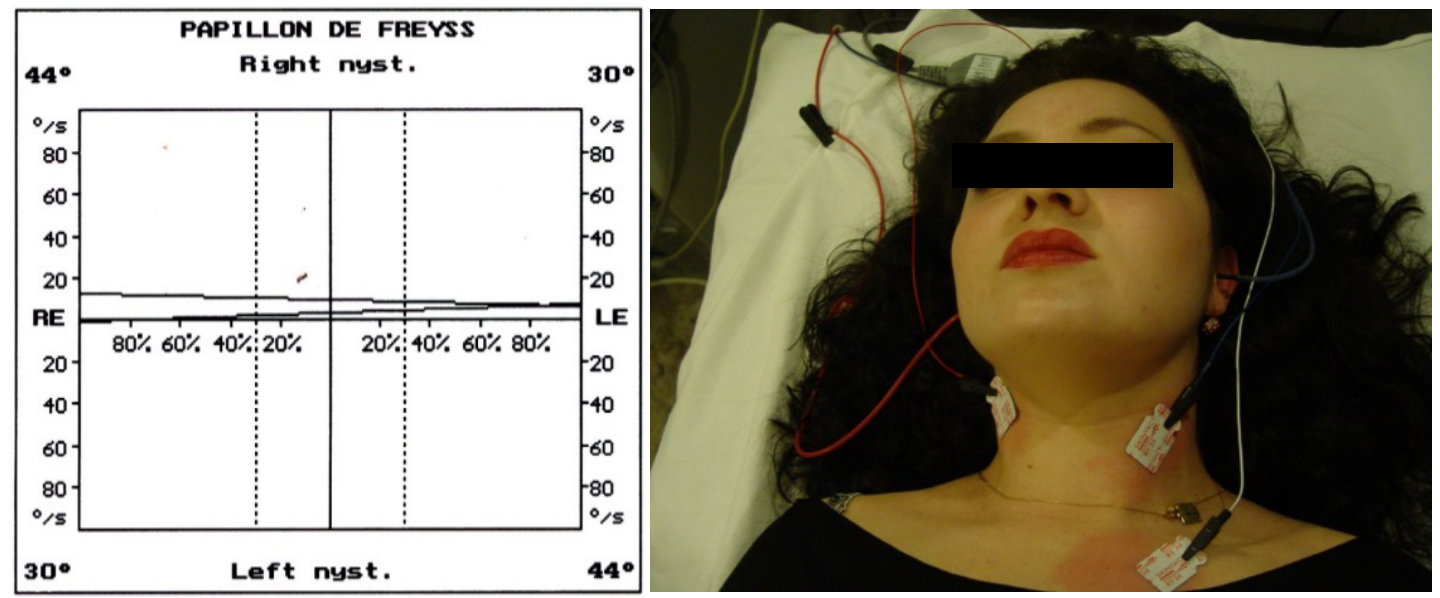

Figure 2b: Objective vestibular test - videonystagmography (caloric test); Left areflexia=lesion of the superior vestibular nerve.

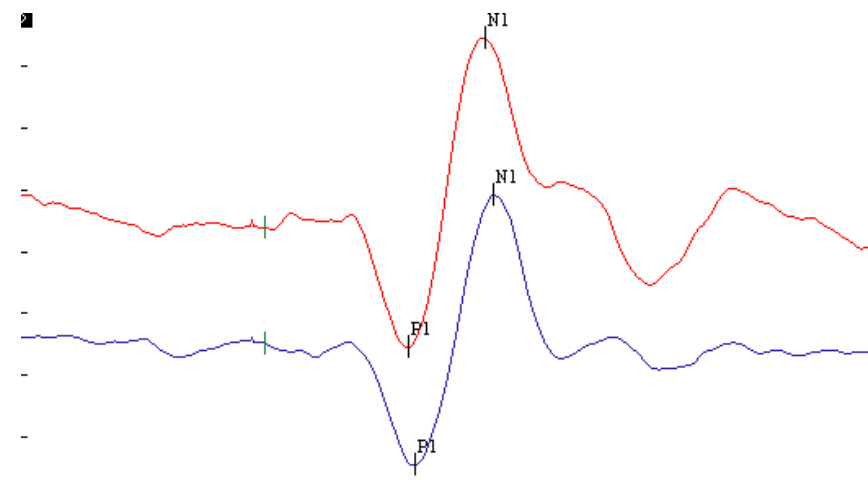

(VEMP) [7.2] Scales: $10.02 \mathrm{~ms} / \mathrm{div}, 100.00 \mathrm{u}$ 'div; $95 \mathrm{~dB} \mathrm{nHL}, 500 \mathrm{~Hz}-\mathrm{A}$, Acc: 150 , Rej: 0 , Rate: 5.1 Normal result in both ears

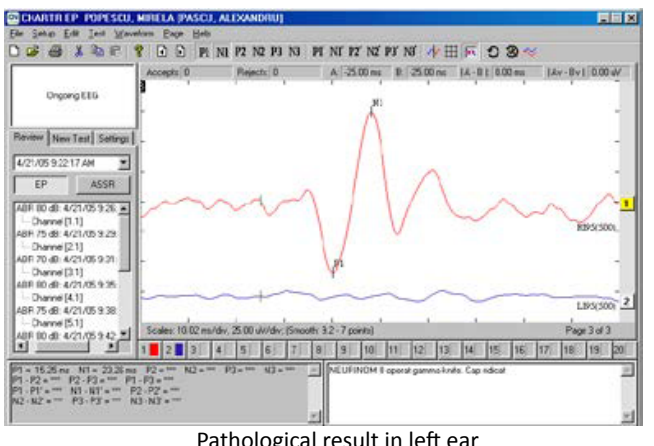

Pathological result in left ear lesion of the inferior vestibular nerve

Figure 2c: Objective vestibular test - Cvemp.

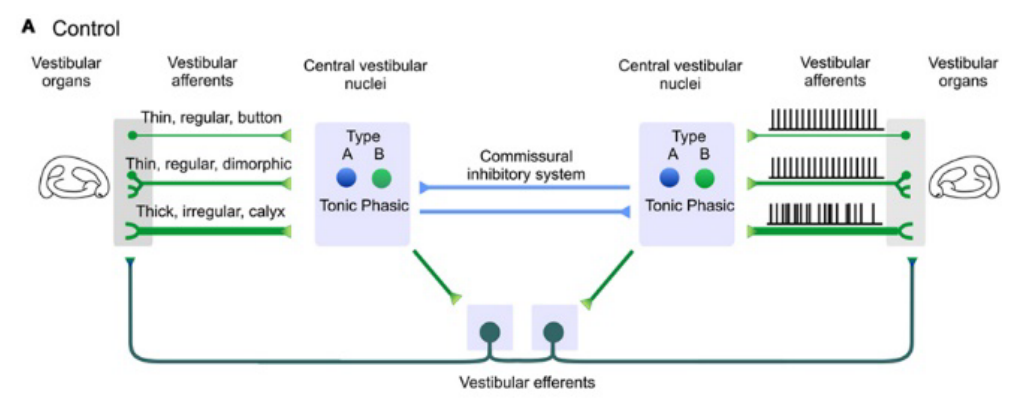

B Compensated after UL

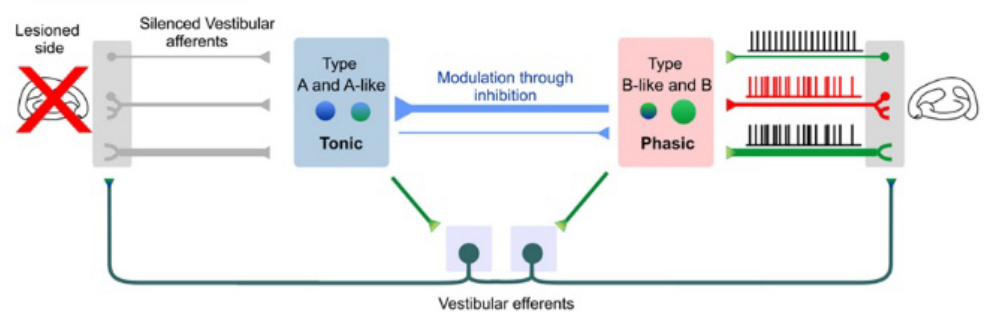

Figure 3: Central vestibular compensation (with Mylan permission). 
Citation: Georgescu M (2017) Vestibular Rehabilitation - Recommended Treatment for Permanent Unilateral Vestibular Loss. Int J Neurorehabilitation 4: 282. doi: 10.4172/2376-0281.1000282

in three categories of functional (F), physical (P) and emotional (E) changes in the quality of life due to vestibular impairment. To these questions the subject can answer with yes/no/sometimes and they are graded with 4, 0 and 2 points, respectively. The total score ranges between 0 and 100: 0-30 indicates mild, 31-60 moderate, and 61-100 severe handicap caused by dizziness [8]. The ABC scale is used for self-assessment of the trust in one's own balance during activities that involve movement (walking around the house, up or down stairs, up or down a ramp, in crowded places, on icy sidewalks, reaching for something, bending over and picking something, getting into or out of a car, stepping onto or off an escalator). Lower ABC scores are associated with lower levels of mobility [9] and falls [10]. ABC scale seems to correlate significantly with DHI in patients with vestibular disorders $[11,12]$.

Recovery assessment is relevant only when considering patient's perspective and not just completing physical performance tests. Questionnaires and balance evaluation methods presented quantify the degree of disability associated with dizziness (DHI), the fear of falling in everyday situations (ABC), the global self-perceived disability (disability scale) and the stability score (SOT)s. They all serve the physician a larger view over the impairment and the way it evolves during treatment.

A customized VR program seems to be the treatment of choice for vestibular neuritis patients, because its effectiveness, safety and lowcost. It improves emotional and functional status and allows resuming independent activities (e.g. driving, walking alone on the street, shopping).

\section{References}

1. Baloh RW (2003) Vestibular neuronitis. N Engl J Med 348: 1027-1032.

2. Strupp M, Brandt T (2010) Vestibular neuritis. In: Eggers SD, Zee D (edr.) Vertigo and imbalance: Clinical neurophysiology of the vestibular system. Handbook of clinical neurophysiology. Elsevier SV, Amsterdam.

3. Mandalà M, Nuti D, Broman AT, Zee DS (2008) Effectiveness of careful bedside examination in assessment, diagnosis and prognosis of vestibular neuritis. Arch Otolaryngol Head Neck Surg 134: 164-169.

4. Lacour M, Sterkers O (2001) Histamine and betahistine in the treatment of vertigo: Elucidation of mechanisms of action. CNS drugs 15: 853-870.

5. Hillier SL, Hollohan V (2007) Vestibular rehabilitation for unilateral peripheral vestibular dysfunction. Cochrane Database Syst Rev 17: CD005397.

6. Kammerlind AS, Ledin TE, Odkvist LM, Skargren EI (2005) Effects of home training and additional physical therapy on recovery after acute unilateral vestibular loss-a randomized study. Clin Rehabil 19: 54-62.

7. Shepard NT, Telian SA, Smith-Wheelock M, Raj A (1993) Vestibular and balance rehabilitation therapy. Ann Otol Rhinol Laryngol 102: 198-205.

8. Cawthorne FS (1944) The physiological basis for head exercises. J Chart Soc Physio Ther 29: 106-107.

9. Cooksey FS (1946) Rehabilitation in vestibular injuries. Proc R Soc Med 39: 273-275.

10. Herdman SJ (2007) Vestibular rehabilitation (Contemporary perspectives in rehabilitation). Davis Company, Philadelphia.

11. Jacobson GP, Newman CW (1990) The development of the dizziness handicap inventory. Arch Otolaryngol Head Neck Surg 116: 424-427.

12. Whitney SL, Wrisley DM, Brown KE, Furman JM (2004) Is perception of handicap related to functional performance in persons with vestibular dysfunction? Otol Neurotol 25: 139-143. 